quatrième série-tome 42 fascicule 5 septembre-octobre 2009

$$
\begin{aligned}
& \text { ANNALES } \\
& \text { SCIENTIFIQUES } \\
& \text { de } \\
& \text { L'ECOLE } \\
& \text { NORMALE } \\
& \text { SUPÉRIEURE }
\end{aligned}
$$

\title{
Gábor SZÉKELYHIDI
}

The Calabi functional on a ruled surface 
Ann. Scient. Éc. Norm. Sup.

$4^{\text {e }}$ série, t. 42,2009, p. 837 à 856

\title{
THE CALABI FUNCTIONAL ON A RULED SURFACE
}

\author{
BY GÁBOR SZÉKELYHIDI
}

\begin{abstract}
We study the Calabi functional on a ruled surface over a genus two curve. For polarizations which do not admit an extremal metric we describe the behavior of a minimizing sequence splitting the manifold into pieces. We also show that the Calabi flow starting from a metric with suitable symmetry gives such a minimizing sequence.

Résumé. - On étudie la fonctionnelle de Calabi sur une surface réglée au-dessus d'une courbe de genre deux. Pour les polarizations qui n'admettent pas de métrique extrémale, on décrit le comportement d'une suite minimisante partitionnant la variété. On montre aussi que le flot de Calabi partant d'une métrique avec une symétrie appropriée produit une telle suite minimisante.
\end{abstract}

\section{Introduction}

In [3] Calabi introduced the problem of minimizing the $L^{2}$-norm of the scalar curvature (this is called the Calabi functional) over metrics in a fixed Kähler class on a compact Kähler manifold. A critical point of the Calabi functional is called an extremal metric. The EulerLagrange equation is that the gradient of the scalar curvature is a holomorphic vector field. It is known that extremal metrics in fact minimize the Calabi functional (see [15], [5], [12]). Recently much progress has been made in understanding when extremal metrics exist, at least on a conjectural level. Kähler-Einstein metrics are a special case and when the first Chern class of the manifold is positive (the manifold is called Fano in this case), Yau conjectured that the existence of Kähler-Einstein metrics is related to the stability of the manifold in the sense of geometric invariant theory. In the case of negative or zero first Chern class Yau [26] and Aubin [2] have shown that Kähler-Einstein metrics always exist, answering a conjecture of Calabi. Tian [24] made significant progress towards understanding the Fano case, solving it completely in the case of surfaces in [23]. Donaldson [9] showed that the scalar curvature can be interpreted as a moment map (this was also observed by Fujiki [13]) and this enabled extending the conjectures about the existence of Kähler-Einstein metrics to more general constant scalar curvature and extremal metrics (see [10], [17], [22]). 
In this paper we look at what we can say about minimizing the Calabi functional in a Kähler class which admits no extremal metric, concentrating on a concrete example. Let $\Sigma$ be a genus 2 curve and $\mathcal{M}$ a degree -1 line bundle on it. We consider the ruled surface $X=\mathbf{P}(\mathcal{M} \oplus \mathcal{O})$ with a family of polarizations $L_{m}=C+m S_{\infty}$, where $C$ is the class of a fibre, $S_{\infty}$ is the infinity section (with self-intersection 1), and $m>0$. Technically we should take $m$ to be rational, especially when discussing test-configurations, but by an approximation and continuity argument we can take $m$ to be real. The aim is to study the problem of minimizing the Calabi functional in these Kähler classes. Our main result is the following.

THEOREM 1. - There exist constants $k_{1} \simeq 18.9, k_{2} \simeq 5.03$, such that

1. If $0<m<k_{1}$ then $X$ admits an extremal metric (this is due to Tonnesen-Friedman [25]).

2. If $k_{1} \leqslant m \leqslant k_{2}\left(k_{2}+2\right)$ then there exists a minimizing sequence of metrics which breaks $X$ into two pieces and converges to complete extremal metrics on both.

3. If $m>k_{2}\left(k_{2}+2\right)$ then there exists a minimizing sequence of metrics which breaks $X$ into three pieces. It converges to complete extremal metrics on two of these and the third degenerates into a fibration of infinitely long and infinitely thin cylinders.

Note that the fact that extremal metrics do not exist in the case $m \geqslant k_{1}$ follows from the work [1] where such existence issues are studied for a large class of ruled manifolds. Alternatively it also follows from Donaldson's theorem [12] on the lower bound of the Calabi functional, and the computations in [22] (see also [21], Section 3.3). Here we go further in that we compute the infimum of the Calabi functional and describe the behavior of a minimizing sequence.

To construct metrics on our ruled surface, we use the momentum construction given in Hwang-Singer [16]. This construction has been used repeatedly in the past to find special metrics on ruled manifolds, in particular extremal metrics. See [1] for a unified treatment of these constructions or [16] for a historical overview and more references. The momentum construction allows us to construct circle invariant metrics from functions on an interval and it gives a convenient expression for the scalar curvature. More precisely, let $\phi:[0, m] \rightarrow \mathbf{R}$ be a smooth function, positive on the interior $(0, m)$, vanishing at the endpoints, and such that $\phi^{\prime}(0)=2, \phi^{\prime}(m)=-2$. The momentum construction gives a metric $\omega_{\phi}$ in the Kähler class $L_{m}$, with scalar curvature

$$
S\left(\omega_{\phi}\right)=\frac{-2}{1+\tau}-\frac{1}{2(1+\tau)}[(1+\tau) \phi]^{\prime \prime} .
$$

Here $\tau$ is the moment map for the $S^{1}$-action on the fibres and working with this coordinate is the central idea of the momentum construction. We will recall this construction in Section 2. Of particular importance to us is the fact that we can consider momentum profiles which vanish on a subset of $(0, m)$. These correspond to degenerate metrics and they arise as the limits of the minimizing sequences in Theorem 1.

In Section 3 we consider the problem of directly minimizing the Calabi functional on the set of metrics obtained by the momentum construction. Since the $L^{2}$-norm of the scalar curvature is equivalent to the $H^{2}$-norm of the momentum profiles, this is straightforward. We find that the Euler-Lagrange equation for a minimizer $\phi$ is $\phi S(\phi)^{\prime \prime}=0$ and $S(\phi)^{\prime \prime}$ must be a negative distribution, i.e., $S(\phi)$ is concave. We show that a unique minimizer exists in each 
Kähler class and its momentum profile is in $C^{2}$. Note that $S(\phi)^{\prime \prime}=0$ is the equation for $\phi$ to define an extremal metric.

In Section 4 we explicitly construct the minimizers, which can be degenerate in the sense that the momentum profiles can vanish on a subset of $(0, m)$. Here we will see the three different kinds of behavior stated in Theorem 1. In Section 5 we construct test-configurations for $X$ and calculate their Futaki invariants. This will clarify the role of the concavity of $S(\phi)$ for minimizers of the Calabi functional. In fact, rational, piecewise-linear convex functions on $[0, m]$ give test-configurations essentially by the construction in [10] as generalized to bundles of toric varieties in [21]. We can approximate $-S(\phi)$ by such functions, and Donaldson's theorem on lower bounds for the Calabi functional in [12] shows that $\omega_{\phi}$ actually achieves the infimum of the Calabi functional on the whole Kähler class, not just the metrics arising from the momentum construction. This will complete the proof of Theorem 1.

An alternative approach to minimizing the Calabi functional is using the Calabi flow introduced in [3]. This is the flow which deforms the Kähler potential in the direction of the scalar curvature. It is expected (see [10], [11]) that the Calabi flow should minimize the Calabi functional and if there is no extremal metric in a given Kähler class, then it should break up the manifold into pieces which admit complete extremal metrics or collapse in some way. In Sections 6 and 7 we will verify this, showing

THEOREM 2. - If the initial metric is given by the momentum construction then the Calabi flow exists for all time and the momentum profiles converge in $\mathrm{H}^{2}$ to the minimizer of the Calabi functional.

Not much is known about the long time behavior of the flow in general, except in the case of Riemann surfaces, where existence and convergence to a uniformizing metric has been shown by Chruściel [8] (see also [4] and [20]). More recently Chen and $\mathrm{He}$ [7] have studied the flow on toric Fano surfaces. The Calabi flow on ruled manifolds has been previously studied in [14], where the long time existence and convergence is proved for the Kähler classes which admit an extremal metric. We use similar techniques, the main difference being that we introduce some variants of the Mabuchi functional when no extremal metric exists. In particular in the unstable case where $k_{1} \leqslant m \leqslant k_{2}\left(k_{2}+2\right)$ we define a functional which decreases along the Calabi flow, is bounded below, and whose derivative is given by the difference between the Calabi functional and its infimum. This leads to the convergence result. The case $m>k_{2}\left(k_{2}+2\right)$ is more delicate since the analogous Mabuchi-type functional is not bounded from below. Nevertheless it has at worst logarithmic decay along the Calabi flow and this is enough to show that the flow minimizes the Calabi functional. This is discussed in Section 6.

As far as the author is aware, Theorem 2 is the first case where the Calabi flow has been successfully analyzed on a manifold which does not admit an extremal metric. We hope that this example will be useful in studying the Calabi flow in general. For example in the Kähler classes which do not admit an extremal metric, we can see that the Sobolev constant and diameter do not remain bounded along the flow. This is in stark contrast to the (normalized) Kähler-Ricci flow, for which the diameter and Sobolev constant remain uniformly bounded for all time (see [19], [27] and [28]). 
Note that throughout the paper we have ignored factors of $2 \pi$, for example in the definition of the Calabi functional. Also we normalize the Futaki invariant slightly differently from usual in Section 5. Hopefully this will lead to no confusion.

Acknowledgements. - Part of this work has appeared in the author's $\mathrm{PhD}$ thesis [21]. I would like to thank my supervisor Simon Donaldson for his encouragement and for sharing his insights.

\section{Metrics on the ruled surface}

In this section we describe the momentum construction for metrics on the ruled surface (see Hwang-Singer [16]). Let $X$ be the ruled surface as above, so that $X=\mathbf{P}(\mathcal{M} \oplus \mathcal{O}) \rightarrow \Sigma$, where $\Sigma$ is a genus 2 curve, and $\mathcal{M}$ is a degree -1 line bundle over $\Sigma$. Let $\omega_{\Sigma}$ be a metric on $\Sigma$ with area $2 \pi$ and constant scalar curvature -2 (we use the "complex" scalar curvature, which is half of the usual Riemannian one). Also, let $h$ be a Hermitian metric on $\mathcal{M}$ with curvature form $i \omega_{\Sigma}$. We consider metrics on $\mathcal{M} \backslash\{0\}$, the complement of the zero section in the total space of $\mathcal{M}$, of the form

$$
\omega=p^{*} \omega_{\Sigma}+2 i \partial \bar{\partial} f(s)
$$

where $p: \mathcal{M} \rightarrow \Sigma$ is the projection map, $s=\frac{1}{2} \log |z|_{h}^{2}$ is the logarithm of the fibrewise norm and $f(s)$ is a suitable strictly convex function that makes $\omega$ positive definite. The point of the momentum construction is the change of coordinate from $s$ to $\tau=f^{\prime}(s)$. The metric $\omega$ is invariant under the $U(1)$-action on $\mathcal{M}$, and $\tau$ is just the moment map for this action. Let $I \subset \mathbf{R}$ be the image of $\tau$, and let $F: I \rightarrow \mathbf{R}$ be the Legendre transform of $f$. By definition this means that

$$
f(s)+F(\tau)=s \tau,
$$

and $F$ is a strictly convex function. The momentum profile is defined to be the function

$$
\phi(\tau)=\frac{1}{F^{\prime \prime}(\tau)} .
$$

We have the following relations:

$$
s=F^{\prime}(\tau), \quad \frac{d s}{d \tau}=F^{\prime \prime}(\tau), \quad \phi(\tau)=f^{\prime \prime}(s) .
$$

The metric in local coordinates. - Let us now see what the metric $\omega$ looks like in local coordinates. Choose a local coordinate $z$ on $\Sigma$ and a fibre coordinate $w$ for $\mathcal{M}$. The fibrewise norm is given by $|(z, w)|_{h}^{2}=|w|^{2} h(z)$ for some positive function $h$, so that

$$
s=\frac{1}{2} \log |w|^{2}+\frac{1}{2} \log h(z)
$$

We can choose the local trivialization of $\mathcal{M}$ in such a way that at a point $\left(z_{0}, w_{0}\right)$ we have $d \log h(z)=0$. We can then compute at the point $\left(z_{0}, w_{0}\right)$

$$
\begin{aligned}
2 i \partial \bar{\partial} f(s) & =i f^{\prime}(s) \partial \bar{\partial} \log h(z)+f^{\prime \prime}(s) \frac{i d w \wedge d \bar{w}}{2|w|^{2}} \\
& =\tau p^{*} \omega_{\Sigma}+\phi(\tau) \frac{i d w \wedge d \bar{w}}{2|w|^{2}} .
\end{aligned}
$$

$4^{\text {e }}$ SÉRIE - TOME $42-2009-\mathrm{N}^{\mathrm{o}} 5$ 
The metric at the point $\left(z_{0}, w_{0}\right)$ is therefore given by

$$
\omega=(1+\tau) p^{*} \omega_{\Sigma}+\phi(\tau) \frac{i d w \wedge d \bar{w}}{2|w|^{2}} .
$$

In order to compute the scalar curvature of $\omega$, note that the determinant of the metric $g$ corresponding to $\omega$ is

$$
\operatorname{det}(g)=\frac{1}{|w|^{2}}(1+\tau) \phi(\tau) \operatorname{det}\left(g_{\Sigma}\right)
$$

which is valid for all points, not just $\left(z_{0}, w_{0}\right)$. The Ricci form at $\left(z_{0}, w_{0}\right)$ is given by

$$
\begin{aligned}
\rho & =-i \partial \bar{\partial} \log \operatorname{det} g \\
& =p^{*} \rho_{\Sigma}-\frac{[(1+\tau) \phi]^{\prime}}{2(1+\tau)} p^{*} \omega_{\Sigma}-\frac{\phi}{2} \cdot \frac{(1+\tau)[(1+\tau) \phi]^{\prime \prime}-[(1+\tau) \phi]^{\prime}}{(1+\tau)^{2}} \cdot \frac{i d w \wedge d \bar{w}}{|w|^{2}},
\end{aligned}
$$

where the derivatives are all with respect to $\tau$ (note that $d / d s=\phi(\tau) d / d \tau$ ) and $\rho_{\Sigma}$ is the Ricci form of the metric $\omega_{\Sigma}$. Taking the trace of this, we find that the scalar curvature of $\omega$ is given by

$$
S(\phi)=\frac{-2}{1+\tau}-\frac{1}{2(1+\tau)}[(1+\tau) \phi]^{\prime \prime}
$$

In [16] the extendability of the metrics across the zero section, and to the projective completion of $\mathcal{M}$ is studied. The proposition we need is the following.

Proposition 3 (see [16]). - For some $m>0$, let $\phi:[0, m] \rightarrow \mathbf{R}$ be a smooth function such that $\phi$ is positive on $(0, m)$, and

$$
\phi(0)=\phi(m)=0, \quad \phi^{\prime}(0)=2, \quad \phi^{\prime}(m)=-2 .
$$

Then the momentum construction defines a smooth metric $\omega_{\phi}$ on $X$ in the Kähler class $C+m S_{\infty}$, with scalar curvature $S(\phi)$ given by Equation 2. Here $C$ is the class of a fibre, and $S_{\infty}$ the infinity section.

If instead $\phi$ satisfies the boundary conditions

$$
\phi(0)=\phi(m)=0, \quad \phi^{\prime}(0)=0, \quad \phi^{\prime}(m)=-2,
$$

and $\phi(\tau) \leqslant O\left(\tau^{2}\right)$ for small $\tau$, then the momentum construction gives a complete metric with finite volume on the complement of the zero section in $X$. Similarly if $\phi^{\prime}(0)=2$ and $\phi^{\prime}(m)=0$ then we obtain a complete metric on the complement of the infinity section.

The metrics are extremal, i.e., their scalar curvature has holomorphic gradient, when $S(\phi)^{\prime \prime}=0$.

Let us also note the definition

Definition 4. - A momentum profile is a $C^{2}$ function $\phi:[0, m] \rightarrow \mathbf{R}$ which is positive on $(0, m)$ and satisfies the boundary conditions (3). A singular momentum profile is the same except we only require it to be non-negative instead of positive, i.e., it can vanish on a subset of $(0, m)$. 
Let us write $\Phi$ for the unique solution of $S(\Phi)^{\prime \prime}=0$ satisfying the same boundary conditions as a momentum profile. Then $\Phi$ is positive on $(0, m)$ precisely when the polarization admits an extremal metric. We define the Calabi functional to be

$$
\begin{aligned}
\operatorname{Cal}(\phi) & =\int_{0}^{m}(S(\phi)-S(\Phi))^{2}(1+\tau) d \tau \\
& =\int_{0}^{m} \frac{1}{4(1+\tau)}\left[((1+\tau)(\Phi-\phi))^{\prime \prime}\right]^{2} d \tau .
\end{aligned}
$$

This differs from the $L^{2}$-norm of $S(\phi)$ by a constant since integrating by parts we get

$$
\int_{0}^{m}(S(\phi)-S(\Phi)) S(\Phi)(1+\tau) d \tau=\int_{0}^{m} \frac{1}{2}[(1+\tau)(\Phi-\phi)]^{\prime \prime} S(\Phi) d \tau=0,
$$

so that

$$
\operatorname{Cal}(\phi)=\int_{0}^{m} S(\phi)^{2}(1+\tau) d \tau-\int_{0}^{m} S(\Phi)^{2}(1+\tau) d \tau .
$$

Throughout the paper when we integrate a function over $X$ which only depends on $\tau$ we will often use the volume form $(1+\tau) d \tau$. From the formula (1) we see that this is a constant multiple of the integral with respect to the volume form $\omega^{2}$. Because of the boundary conditions on $\phi$ the Poincare inequality shows that the Calabi functional is equivalent to the $H^{2}$-norm of $\phi$. This makes it easy to minimize the Calabi functional directly as we do in the next section.

\section{Minimising the Calabi functional}

It is fairly simple to directly minimize the Calabi functional on the set of metrics which are given by momentum profiles. We introduce the set of functions

$$
A=\left\{\begin{array}{l|l}
\phi:[0, m] \rightarrow \mathbf{R} & \begin{array}{l}
\phi \in H^{2}, \phi \geqslant 0 \text { and } \phi \text { satisfies the } \\
\text { boundary conditions (3) in Proposition 3 }
\end{array}
\end{array}\right\},
$$

and we want to minimize the Calabi functional on this space. Let us choose a minimizing sequence $\phi_{k} \in A$. Writing $G_{k}=(1+\tau)\left(\phi_{k}-\Phi\right)$, we see from Equation (4) that we have a bound $\left\|G_{k}^{\prime \prime}\right\|_{L^{2}} \leqslant C_{1} \cdot \operatorname{Cal}\left(\phi_{k}\right)$. Since $G_{k}$ and $G_{k}^{\prime}$ vanish at the endpoints of $[0, m]$, using the Poincaré inequality we obtain a bound $\left\|\left(\phi_{k}-\Phi\right)^{\prime \prime}\right\|_{L^{2}} \leqslant C_{2} \cdot \operatorname{Cal}\left(\phi_{k}\right)$. Using the Poincaré inequality again, this implies a bound $\left\|\phi_{k}-\Phi\right\|_{H^{2}} \leqslant C_{3} \cdot \operatorname{Cal}\left(\phi_{k}\right)$, so since $\Phi$ is fixed, we have $\left\|\phi_{k}\right\|_{H^{2}} \leqslant C \cdot \operatorname{Cal}\left(\phi_{k}\right)$. Therefore we can choose a subsequence converging weakly to some $\phi \in H^{2}$. Weak convergence in $H^{2}$ implies convergence in $C^{1}$ so the boundary conditions and non-negativity hold in the limit, i.e., $\phi \in A$. Moreover $\mathrm{Cal}$ is lower-semicontinuous because the $H^{2}$-norm is, so $\phi$ is the required minimizer.

Proposition 5. - The minimizer $\phi$ in A satisfies $\phi S(\phi)^{\prime \prime}=0$ and $S(\phi)^{\prime \prime}$ is a negative distribution. In particular $S(\phi)$ is continuous, so $\phi \in C^{2}$. Conversely if $\psi S(\psi)^{\prime \prime}=0$ and $S(\psi)$ is concave, then $\psi=\phi$. 
Proof. - The variation of $C a l$ at $\phi$ is given by

$$
\operatorname{DCal}_{\phi}(\widetilde{\phi})=-\int_{0}^{m}(S(\phi)-S(\Phi))[(1+\tau) \widetilde{\phi}]^{\prime \prime} d \tau .
$$

We are considering variations inside $A$, so $\widetilde{\phi}$ and its first derivative vanish at the endpoints. We can therefore integrate by parts, and find that

$$
-\int_{0}^{m} S(\phi)^{\prime \prime} \widetilde{\phi}(1+\tau) d \tau \geqslant 0
$$

for all $\widetilde{\phi}$ such that $\phi+\epsilon \widetilde{\phi} \in A$ for small enough $\epsilon$. We can choose $\widetilde{\phi}$ to be an arbitrary non-negative smooth function which vanishes along with its first derivative at the endpoints. This shows that $S(\phi)^{\prime \prime}$ is a negative distribution. On the open set where $\phi$ is positive we can choose $\widetilde{\phi}$ to be negative or positive, so it follows that $S(\phi)^{\prime \prime}=0$ at these points. Therefore $\phi S(\phi)^{\prime \prime}=0$ on $(0, m)$. The continuity of $S(\phi)$ follows from it being concave, and this implies that $\phi \in C^{2}$.

The converse follows from the following computation.

$$
\begin{aligned}
\operatorname{Cal}(\psi) & \leqslant \operatorname{Cal}(\psi)+\int_{0}^{m}(S(\phi)-S(\psi))^{2}(1+\tau) d \tau \\
& =\operatorname{Cal}(\phi)+2 \int_{0}^{m}(S(\psi)-S(\phi)) S(\psi)(1+\tau) d \tau \\
& =\operatorname{Cal}(\phi)+\int_{0}^{m}[(1+\tau) \phi-(1+\tau) \psi]^{\prime \prime} S(\psi) d \tau \\
& =\operatorname{Cal}(\phi)+\int_{0}^{m} \phi S(\psi)^{\prime \prime}(1+\tau) d \tau \\
& \leqslant \operatorname{Cal}(\phi) .
\end{aligned}
$$

Since $\operatorname{Cal}(\phi)$ is minimal we must have equality, i.e.,

$$
\int_{0}^{m}(S(\phi)-S(\psi))^{2}(1+\tau) d \tau=0
$$

This implies that $S(\phi)=S(\psi)$, from which it follows that $\phi=\psi$.

\section{Explicit minimizers}

In this section we compute explicitly the minimizers of the Calabi functional for all polarizations. For each $m$ we are looking for a singular momentum profile (Definition 4 ) such that $S(\phi)^{\prime \prime}=0$ wherever $\phi$ does not vanish, and in addition $S(\phi)$ is concave.

There are three cases to consider depending on the polarization.

Case 1. There exists an extremal metric, $m<k_{1} \simeq 18.889$. - In this case we want to solve the equation $S(\phi)^{\prime \prime}=0$. By the Formula (2) for the scalar curvature, this is the ODE

$$
\frac{1}{2(1+\tau)}\left(-4-[(1+\tau) \phi]^{\prime \prime}\right)=A \tau+B
$$

for some constants $A, B$. Rearranging this and integrating twice we obtain

$$
(1+\tau) \phi=-\frac{A \tau^{4}}{6}-\frac{(A+B) \tau^{3}}{3}-B \tau^{2}-2 \tau^{2}+C \tau+D
$$


where $C$ and $D$ are also constants. The boundary conditions on $\phi$ on the interval $[0, m]$ give a system of linear equations on $A, B, C, D$ which we can solve to obtain

$$
\phi(\tau)=\frac{2 \tau(m-\tau)}{m\left(m^{2}+6 m+6\right)(1+\tau)}\left[\tau^{2}(2 m+2)+\tau\left(-m^{2}+4 m+6\right)+m^{2}+6 m+6\right] .
$$

This will give a metric when it is positive on the interval $(0, m)$ which happens if and only if the quadratic expression in square brackets is positive on this interval. This is the case for $m<k_{1}$ where $k_{1}$ is the only positive real roof of the quartic $m^{4}-16 m^{3}-52 m^{2}-48 m-12$. Approximately $k_{1} \simeq 18.889$, which is the result obtained by Tønessen-Friedman [25]. See Figure 1 for a graph of $\phi(\tau)$ for $m=17$.

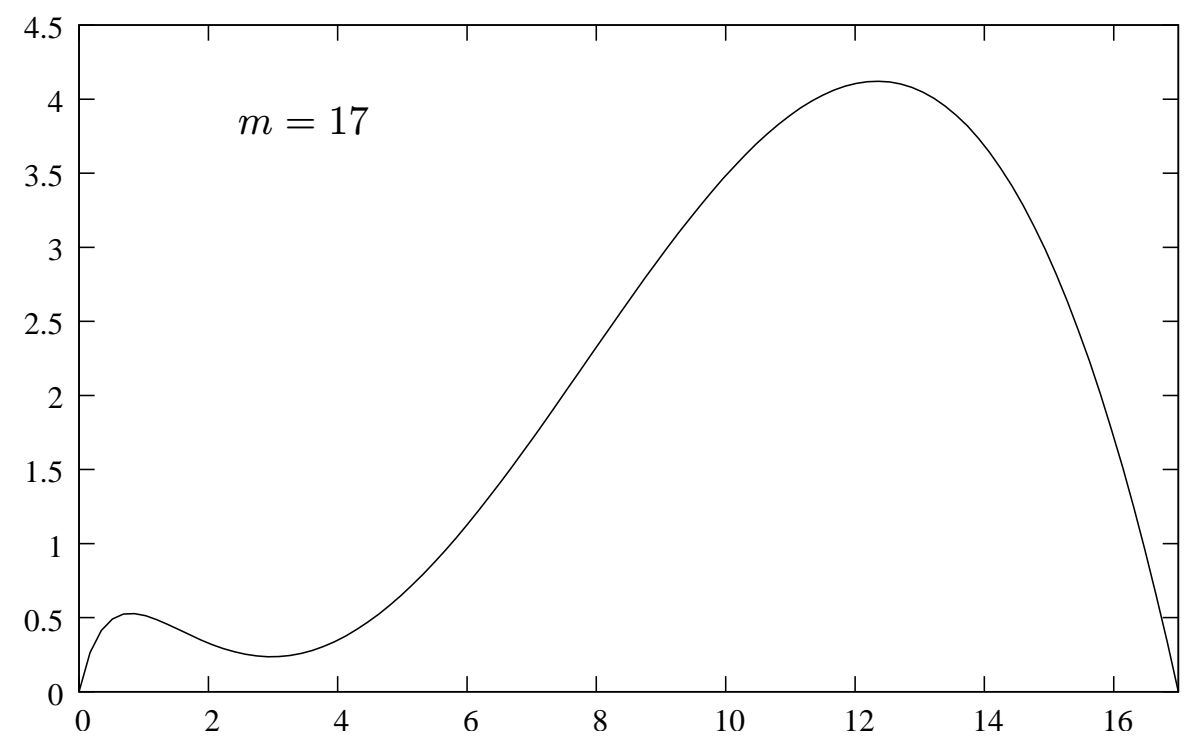

Figure 1. Momentum profile of an extremal metric on $X$ when $m=17$.

Case 2. $X$ breaks up into two pieces, $k_{1} \leqslant m \leqslant k_{2}\left(k_{2}+2\right) \simeq 35.33$. - When $m \geqslant k_{1}$ we can no longer find a positive solution of $S(\phi)^{\prime \prime}=0$ on the whole interval $[0, m]$ so we split the interval into two pieces $[0, c]$ and $[c, m]$. We would like to find $\phi$ which vanishes at $c$, but on the intervals $(0, c)$ and $(c, m)$ we have $S(\phi)^{\prime \prime}=0$, and $S(\phi)$ is concave on $[0, m]$. We first let $\phi_{1}$ be the solution of the equation

$$
\begin{gathered}
S\left(\phi_{1}\right)^{\prime \prime}=0 \text { on the interval }(0, c) \\
\phi_{1}(0)=\phi_{1}(c)=0, \quad \phi_{1}^{\prime}(0)=2, \quad \phi_{1}^{\prime}(c)=0 .
\end{gathered}
$$

We obtain

$$
\phi_{1}(\tau)=\frac{2 \tau(c-\tau)^{2}}{c^{2}\left(c^{2}+6 c+6\right)(1+\tau)}\left[\tau\left(-c^{2}+2 c+3\right)+c^{2}+6 c+6\right]
$$

This is positive on $(0, c)$ if the linear expression in square brackets is positive on this interval. This happens for $c \leqslant k_{2}$ where $k_{2}$ is the only positive real root of the cubic $c^{3}-3 c^{2}-9 c-6$. 
Approximately $k_{2} \simeq 5.0275$. The scalar curvature is given by

$$
S\left(\phi_{1}\right)=\frac{12\left(c^{2}-2 c-3\right)}{c^{2}\left(c^{2}+6 c+6\right)} \tau-\frac{6\left(2 c^{2}-c-4\right)}{c\left(c^{2}+6 c+6\right)} .
$$

To deal with the interval $[c, m]$ we first solve the equation

$$
\begin{gathered}
S(\psi)^{\prime \prime}=0 \text { on the interval }(0, d) \\
\psi(0)=\psi(d)=0, \quad \psi^{\prime}(0)=0, \quad \psi^{\prime}(d)=-2
\end{gathered}
$$

for some constant $d$, and then shift the solution to $[c, m]$. The solution on $[0, d]$ is given by

$$
\psi(\tau)=\frac{2 \tau^{2}(d-\tau)}{d^{2}\left(d^{2}+6 d+6\right)(1+\tau)}\left[\tau\left(2 d^{2}+4 d+3\right)-d^{3}+3 d^{2}+9 d+6\right] .
$$

As before, this is positive on $(0, d)$ if the linear term in square brackets is positive on this interval. This is the case for $d \leqslant k_{2}$, for the same $k_{2}$ as above. The scalar curvature is given by

$$
S(\psi)=\frac{12\left(2 d^{2}+4 d+3\right)}{d^{2}\left(d^{2}+6 d+6\right)} \tau-\frac{6\left(3 d^{2}+5 d+2\right)}{d\left(d^{2}+6 d+6\right)}
$$

Now note that if we define $\phi_{2}$ by

$$
\phi_{2}(\tau)=(c+1) \psi\left(\frac{\tau-c}{c+1}\right)
$$

then $\phi_{2}$ solves the equation

$$
\begin{gathered}
S\left(\phi_{2}\right)^{\prime \prime}=0 \text { on the interval }(c,(c+1) d+c) \\
\phi_{2}(c)=\phi_{2}((c+1) d+c)=0, \quad \phi_{2}^{\prime}(c)=0, \quad \phi_{2}^{\prime}((c+1) d+c)=-2 .
\end{gathered}
$$

The scalar curvature is given by

$$
S\left(\phi_{2}\right)(\tau)=\frac{1}{c+1} S(\psi)\left(\frac{\tau-c}{c+1}\right)
$$

We now define $\phi$ by

$$
\phi(\tau)= \begin{cases}\phi_{1}(\tau) & \tau \in[0, c], \\ \phi_{2}(\tau) & \tau \in[c,(c+1) d+c] .\end{cases}
$$

We can check that $S(\phi)$ will be continuous at $\tau=c$ precisely when $c=d$. We also want $(c+1) d+c=m$, which implies that $c=\sqrt{m+1}-1$. With these choices a simple computation shows that $S(\phi)$ is concave for $m \geqslant k_{1}$ (note that it is linear for $m=k_{1}$, and convex for $\left.m<k_{1}\right)$. Finally recall that the condition that $\phi$ is non-negative means that $c \leqslant k_{2}$, which in turn implies $m \leqslant k_{2}\left(k_{2}+2\right)$. See Figure 2 for a graph of $\phi$ for $m=24$. 


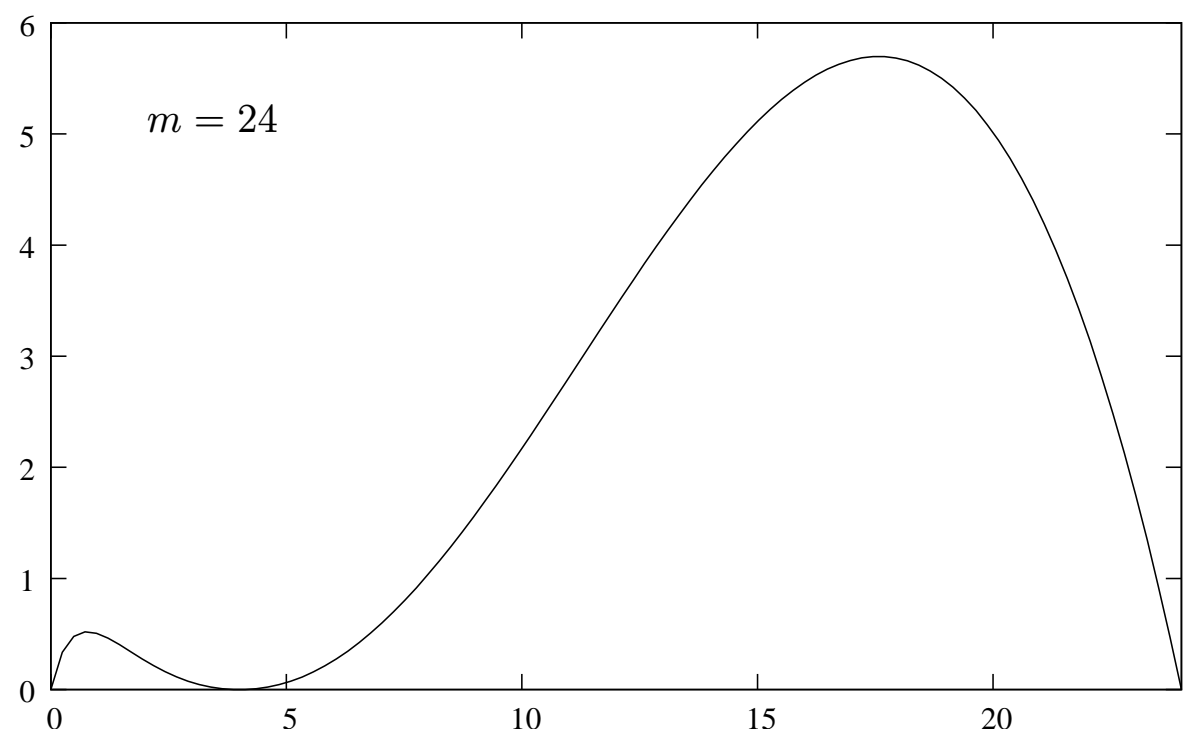

Figure 2. Momentum profile of the minimizer on $X$ when $m=24$. The manifold breaks into two pieces both of which are equipped with a complete extremal metric.

Case 3. $X$ breaks up into three pieces, $m>k_{2}\left(k_{2}+2\right)$. - The previous construction no longer works for $m>k_{2}\left(k_{2}+2\right)$ so we need to split the interval $[0, m]$ into three pieces. From the previous case we have a solution $\phi_{1}$ to the equation

$$
\begin{gathered}
S\left(\phi_{1}\right)^{\prime \prime}=0 \text { on the interval }\left(0, k_{1}\right) \\
\phi_{1}(0)=\phi_{1}\left(k_{1}\right)=0, \quad \phi_{1}^{\prime}(0)=2, \quad \phi_{1}^{\prime}\left(k_{1}\right)=0,
\end{gathered}
$$

and also a solution $\phi_{2}$ to

$$
\begin{aligned}
S\left(\phi_{2}\right)^{\prime \prime} & =0 \text { on the interval }(c, m) \\
\phi_{2}(c)=\phi_{2}(m) & =0, \quad \phi_{2}^{\prime}(c)=0, \quad \phi_{2}^{\prime}(m)=-2,
\end{aligned}
$$

where the constant $c$ is defined by

$$
c=\frac{m+1}{k_{2}+1}-1
$$

We define

$$
\phi(\tau)= \begin{cases}\phi_{1}(\tau) & \tau \in\left[0, k_{2}\right] \\ 0 & \tau \in\left[k_{2}, c\right] \\ \phi_{2}(\tau) & \tau \in[c, m] .\end{cases}
$$

We can check that $c>k_{2}$ precisely when $m>k_{2}\left(k_{2}+2\right)$, and this choice of $\phi$ satisfies that $\phi S(\phi)^{\prime \prime}=0$ and $S(\phi)$ is concave. See Figure 3 for a graph of $\phi$ for $m \simeq 41.2$.

Conclusion. - For any $m$ one of the previous 3 cases will hold, so we can construct a $\phi$ which satisfies the equation $\phi S(\phi)^{\prime \prime}=0$ and $S(\phi)$ is concave. According to Proposition 5 this $\phi$ will give the minimum of the Calabi functional on the space of singular momentum profiles. In 


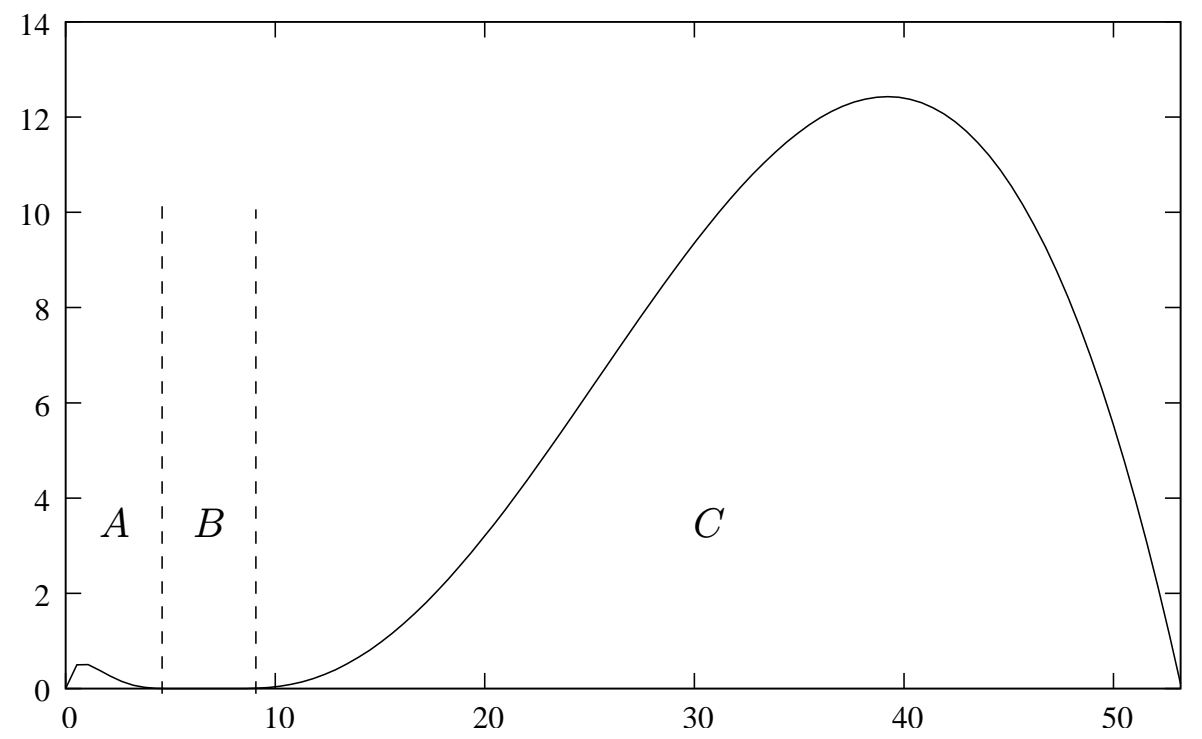

Figure 3. Momentum profile of the minimizer on $X$ when $m \simeq 53.2$. The manifold breaks into three pieces, two of which, $A$ and $C$, admit complete extremal metrics, and in the third, $B$, the $S^{1}$-orbits collapse.

the next section we will show that they give the infimum of the Calabi functional over all metrics in their Kähler class. This will complete the proof of Theorem 1.

\section{Test-configurations}

In the previous section we have found a (possibly degenerate) metric in each Kähler class, which minimizes the Calabi functional on the set of metrics which come from the momentum construction. In this section we want to show that these metrics minimize the Calabi functional on their entire Kähler class. For this we use Donaldson's theorem [12] which gives a lower bound on the Calabi functional, given a destabilizing test-configuration. We will not give a detailed explanation of the test-configurations that we use, and the computation of their Futaki invariants. For more details see [21] and [10].

Proposition 6 (Donaldson [12]). - Suppose there exists a test-configuration $\chi$ for a polarized variety $(X, L)$ such that the Futaki invariant $F(\chi)$ is negative. Then for any metric $\omega$ in the class $c_{1}(L)$ we have the inequality

$$
\|S(\omega)-\hat{S}\|_{L^{2}} \geqslant \frac{-F(\chi)}{\|\chi\|} .
$$

Recall that a test-configuration for $(X, L)$ is a flat $\mathbf{C}^{*}$-equivariant family $\mathcal{X} \rightarrow \mathbf{C}$ with an equivariant ample line bundle $\mathcal{L}$, such that for $t \neq 0$ the fibre $\mathcal{X}_{t}$ is isomorphic to $X$, and $\left.\mathcal{L}\right|_{\mathcal{X}_{t}}=L^{r}$ for some integer $r>0$. Given a test-configuration $\chi$ one defines the Futaki invariant $F(\chi) \in \mathbf{R}$ by looking at the weights of the induced $\mathbf{C}^{*}$-action on the central fibre. 
For details see [10], and for the definition of the norm $\|\chi\|$ see [12]. Also note that we have ignored factors of $2 \pi$.

In order to show that in each Kähler class the degenerate metric $\omega$ we found in the previous section minimizes the Calabi functional, we want to define a sequence of test-configurations $\chi_{i}$ for which

$$
\lim _{i \rightarrow \infty} \frac{-F\left(\chi_{i}\right)}{\left\|\chi_{i}\right\|}=\|S(\omega)-\hat{S}\|_{L^{2}}
$$

Using Donaldson's theorem this implies that this is the infimum of the Calabi functional. To obtain test-configurations we use the construction in [21] Section 4.1 (Theorem 4.1.2), which is an extension of the construction of test-configurations for toric varieties by Donaldson [10] to bundles of toric varieties. For the case of our ruled surface we obtain

Proposition 7. - Given a rational, piecewise-linear, convex function $h:[0, m] \rightarrow \mathbf{R}$, there exists a test-configuration for $\left(X, L_{m}\right)$ with Futaki invariant given by

$$
F(h)=h(0)+(1+m) h(m)-2 \int_{0}^{m} h(\tau) d \tau-\hat{S} \int_{0}^{m} h(\tau)(1+\tau) d \tau
$$

and norm

$$
\|h\|^{2}=\int_{0}^{m}(h(\tau)-\hat{h})^{2}(1+\tau) d \tau,
$$

where $\hat{h}$ is the average of $h$ with respect to the measure $(1+\tau) d \tau$.

We now briefly describe what these test-configurations look like. On each $\mathbf{P}^{1}$ fibre they are given by Donaldson's construction of test-configurations on toric varieties. The central fibre is a chain of normal crossing $\mathbf{P}^{1}$ 's, one for each linear piece of $h$, and the $\mathbf{C}^{*}$-action on each piece is determined by the slope of $h$. In our situation this is done over a base curve, so the central fibre will be a chain $X_{1}, \ldots, X_{k}$ of copies of $X$, so that the infinity section of $X_{i}$ is glued to the zero section of $X_{i+1}$. In particular when $h$ is only non-linear at one point, then the test-configuration is essentially a deformation to the normal cone of the zero section of $X$ (see [18] for the definition of deformation to the normal cone), except the $\mathbf{C}^{*}$-action on the total space of the test-configuration is multiplied by a $\mathbf{C}^{*}$-action lifted from $X$.

To work with test-configurations we should restrict to polarizations $L_{m}$ with $m$ rational but an approximation argument gives us the conclusion of Proposition 6 for any real $m$ as well. Given a continuous convex function $h$ on $[0, m]$ which is not necessarily rational or piecewise-linear, we still define the "Futaki invariant" $F(h)$ of $h$ by Equation 7.

Lemma 8. - Let $\phi$ be a singular momentum profile, and $h:[0, m] \rightarrow \mathbf{R}$ a piecewise-smooth convex function. Suppose that $h$ is linear on any interval on which $\phi$ does not vanish identically. Then

$$
F(h)=\int_{0}^{m} h(\tau)(S(\phi)-\hat{S})(1+\tau) d \tau
$$

Proof. - The proof is a simple integration by parts, using the formulas for $F(h)$ and $S(\phi)$. 
This result is analogous to the fact that the Futaki invariant of a holomorphic vector field can be computed algebro-geometrically or differential geometrically (see [10]). Here if $h$ is rational and piecewise-linear then it does not define a holomorphic vector field but the result says that we can still compute the Futaki invariant of the test-configuration it induces with a differential geometric formula as long as we use a metric which degenerates in a suitable way at points where $h$ is not linear.

We can now complete the proof of Theorem 1.

Proof of Theorem 1. - What remains to be shown is that for each polarization, the minimizer $\phi$ that we have constructed in the previous section minimizes the Calabi functional over the whole Kähler class, not just over the set of metrics obtained from the momentum construction. Let $\phi$ be one of these minimizers. Since $-S(\phi)$ is convex, we can approximate it in the $C^{0}$-norm by a sequence of rational, piecewise-linear convex functions $h_{i}$. These define a sequence of test-configurations $\chi_{i}$ such that

$$
\lim _{i \rightarrow \infty} \frac{-F\left(\chi_{i}\right)}{\left\|\chi_{i}\right\|}=\frac{-F(-S(\phi))}{\|S(\phi)-\hat{S}\|_{L^{2}}} .
$$

If we let $h=-S(\phi)$, then $\phi$ and $h$ satisfy the conditions of Lemma 8 so that

$$
F(-S(\phi))=-\int_{0}^{m} S(\phi)(S(\phi)(\tau)-\hat{S})(1+\tau) d \tau=-\|S(\phi)-\hat{S}\|_{L^{2}}^{2} .
$$

Therefore

$$
\lim _{i \rightarrow \infty} \frac{-F\left(\chi_{i}\right)}{\left\|\chi_{i}\right\|}=\|S(\phi)-\hat{S}\|_{L^{2}}
$$

so that Proposition 6 now implies that this limit is the infimum of the Calabi functional on the Kähler class.

\section{The Calabi flow}

We have seen that in the case of a ruled surface it is fairly simple to minimize the Calabi functional directly over the set of metrics given by momentum profiles. It is also interesting to see whether the Calabi flow converges to these minimizers. In this section we will prove that this is the case. In [14] Guan has shown that on a ruled manifold when an extremal metric exists, then starting from a metric given by the momentum construction the Calabi flow exists for all time and converges to the extremal metric exponentially fast. Our techniques are similar to his, but we need to introduce some new functionals which are modifications of the Mabuchi functional more suited for studying the unstable polarizations.

We consider a family of metrics $\omega_{s}$ given by the momentum construction (see Section 2), i.e., on the complement of the zero and infinity sections we have

$$
\omega_{t}=p^{*} \omega_{\Sigma}+2 i \partial \bar{\partial} f_{t}(s),
$$

for some family of suitably convex functions $f_{t}$. This path of metrics satisfies the Calabi flow if

$$
\frac{\partial f_{t}}{\partial t}=S\left(\omega_{t}\right)
$$


If we denote by $F_{t}$ the Legendre transforms of the $f_{t}$, then from the definition of the Legendre transformation we find

$$
\frac{\partial F_{t}}{\partial t}=-\frac{\partial f_{t}}{\partial t}
$$

so that the path of momentum profiles $\phi_{t}=1 / F_{t}^{\prime \prime}$ satisfies

$$
\frac{\partial \phi_{t}}{\partial t}=\phi_{t}^{2} S\left(\phi_{t}\right)^{\prime \prime}
$$

where $S\left(\phi_{t}\right)$ is given by Equation 2. Note that $\phi_{t}$ only determines $f_{t}$ up to translation and adding a constant. Therefore Equation (8) is the Calabi flow up to holomorphic automorphisms.

It is known that the flow exists for a short time with any smooth initial metric (see Chen$\mathrm{He}[6])$. Also, the Calabi functional is decreased under the flow:

Lemma 9. - If $\phi$ is a solution to the Calabiflow, then

$$
\frac{d \operatorname{Cal}(\phi)}{d t}=-\int_{0}^{m} \phi^{2}\left(S(\phi)^{\prime \prime}\right)^{2}(1+\tau) d \tau \leqslant 0 .
$$

In particular the $H^{2}$ norm of $\phi_{t}$ is uniformly bounded along the flow.

Proof. - The result follows from the following computation of the variation.

$$
\begin{aligned}
\frac{d \operatorname{Cal}(\phi)}{d t} & =2 \int_{0}^{m}(S(\phi)-S(\Phi))\left(-\frac{1}{2(1+\tau)}\left[(1+\tau) \phi^{2} S(\phi)^{\prime \prime}\right]^{\prime \prime}\right)(1+\tau) d \tau \\
& =-\int_{0}^{m} \phi^{2}\left(S(\phi)^{\prime \prime}\right)^{2}(1+\tau) d \tau .
\end{aligned}
$$

We can perform the integration by parts because $\phi^{2}$ and $\left(\phi^{2}\right)^{\prime}$ vanish at the endpoints. Also recall that $S(\Phi)^{\prime \prime}=0$.

In Section 7 we will show that there is a solution to the Calabi flow for all time for any polarization. In this section we concentrate on proving the following.

Proposition 10. - If the flow exists for all time then the momentum profiles converge in $H^{2}$ to the minimizer that we found in Section 4.

Proof. - Let us write $\Psi$ for the minimizer, so when $m<k_{1}$ then $\Psi$ is the momentum profile of an extremal metric, when $m \leqslant m \leqslant k_{2}\left(k_{2}+2\right)$ then $\Psi$ vanishes at an interior point of $(0, m)$ and when $m>k_{2}\left(k_{2}+2\right)$ then $\Psi$ vanishes on an interval inside $(0, m)$.

Introduce the functional

$$
\mathcal{M}(\phi)=\int_{0}^{m}\left(\frac{\Psi}{\phi}+\log \phi\right)(1+\tau) d \tau
$$

defined on momentum profiles $\phi$. When $m<k_{1}$ then in fact $\mathcal{M}$ is the modified Mabuchi functional (see [1] Section 2.3). 
The key point is that $\mathcal{M}$ is decreasing under the flow (this is well-known for the modified Mabuchi functional, since the Calabi flow is its gradient flow). This follows from the computation

$$
\begin{aligned}
\frac{d \mathcal{M}\left(\phi_{t}\right)}{d t} & =\int_{0}^{m}\left(-\Psi S\left(\phi_{t}\right)^{\prime \prime}+\phi_{t} S\left(\phi_{t}\right)^{\prime \prime}\right)(1+\tau) d \tau \\
& =\int_{0}^{m}\left(\phi_{t}-\Psi\right)\left(S\left(\phi_{t}\right)-S(\Psi)\right)^{\prime \prime}(1+\tau) d \tau+\int_{0}^{m} \phi_{t} S(\Psi)^{\prime \prime}(1+\tau) d \tau \\
& \leqslant-2 \int_{0}^{m}\left(S\left(\phi_{t}\right)-S(\Psi)\right)^{2}(1+\tau) d \tau
\end{aligned}
$$

where we have used that $\Psi S(\Psi)^{\prime \prime}=0$ and $S(\Psi)^{\prime \prime}$ is a negative distribution.

On the other hand we have that

$$
\mathcal{M}(\phi) \geqslant \int_{0}^{m} \log \phi \cdot(1+\tau) d \tau \geqslant-C_{1} \int_{0}^{m} \log \frac{\Theta}{\phi} d \tau-C_{2},
$$

where $\Theta$ is a fixed momentum profile and $C_{1}, C_{2}$ are constants. Since log is concave we obtain

$$
\mathcal{M}(\phi) \geqslant-C_{3} \log \int_{0}^{m} \frac{\Theta}{\phi} d \tau-C_{4},
$$

for some constants $C_{3}, C_{4}$. The lemma that follows now implies that along the flow

$$
\mathcal{M}\left(\phi_{t}\right) \geqslant-C \log (1+t)-D
$$

Since $\mathcal{M}\left(\phi_{t}\right)$ is decreasing, we necessarily have that along a subsequence its derivative tends to zero, i.e., $S\left(\phi_{t}\right) \rightarrow S(\Psi)$ in $L^{2}$ (integrating with respect to $(1+\tau) d \tau$ as usual). Since $\left\|S\left(\phi_{t}\right)\right\|_{L^{2}}$ is decreasing along the flow, it follows that

$$
\lim _{t \rightarrow \infty}\left\|S\left(\phi_{t}\right)\right\|_{L^{2}}=\|S(\Psi)\|_{L^{2}} .
$$

Let us now take any subsequence $\phi_{i}$. Because of the uniform $H^{2}$-bound there is a subsequence also denoted by $\phi_{i}$ which converges weakly in $H^{2}$ to some limit. Now Equation 10 implies the convergence of the $H^{2}$-norms, which together with the weak convergence implies strong convergence in $H^{2}$. The limit then has to be $\Psi$ since the minimizer of the Calabi functional is unique (Proposition 5).

Lemma 11. - Let $\Theta:[0, m] \rightarrow \mathbf{R}$ be a momentum profile. For the solution $\phi_{t}$ to the Calabi flow we have

$$
\int_{0}^{m} \frac{\Theta}{\phi_{t}} d \tau<C(1+t)
$$

for some constant $C$.

Proof. - Let us define the functional

$$
\mathcal{F}(\psi)=\int_{0}^{m} \frac{\Theta}{\psi}-\log \frac{\Theta}{\psi} d \tau
$$


for any momentum profile $\psi$. Along the Calabi flow we have

$$
\begin{aligned}
\frac{d}{d t} \mathcal{F}\left(\phi_{t}\right) & =\int_{0}^{m}\left(\phi_{t}-\Theta\right) S\left(\phi_{t}\right)^{\prime \prime} d \tau=\int_{0}^{m}\left(\phi_{t}-\Theta\right)^{\prime \prime} S\left(\phi_{t}\right) d \tau \\
& \leqslant\left(\int_{0}^{m}\left(\phi_{t}^{\prime \prime}-\Theta^{\prime \prime}\right)^{2} d \tau\right)^{1 / 2}\left(\operatorname{Cal}\left(\phi_{t}\right)+C\right)^{1 / 2}
\end{aligned}
$$

The uniform $H^{2}$ bound on $\phi_{t}$ now implies that $\mathcal{F}\left(\phi_{t}\right) \leqslant C(1+t)$ for some $C>0$. The result follows from the inequality $x-\log x>x / 2$.

REMARK. - Note that when $m \leqslant k_{2}\left(k_{2}+2\right)$ the functional $\mathcal{M}$ is bounded below on the set of momentum profiles. This is because we can write

$$
\mathcal{M}(\phi)=\int_{0}^{m}\left(\frac{\Psi}{\phi}-\log \frac{\Psi}{\phi}\right)(1+\tau) d \tau+\int_{0}^{m} \log \Psi \cdot(1+\tau) d \tau .
$$

Since $\Psi$ only vanishes at isolated points and to finite order, the integral of $\log \Psi$ is finite, so the inequality $\log x<x$ implies

$$
\mathcal{M}(\phi) \geqslant \int_{0}^{m} \log \Psi \cdot(1+\tau) d \tau .
$$

In the case $m>k_{2}\left(k_{2}+2\right)$ however $\mathcal{M}$ is not bounded from below since now $\Psi$ vanishes on an interval. In particular as $\phi \rightarrow \Psi$, it is clear that $\mathcal{M}(\phi) \rightarrow-\infty$.

\section{Long time existence}

The existence of the Calabi flow for a short time has been proved by Chen-He [6] (also Guan [14] for ruled manifolds). In the case when an extremal metric exists, the long time existence has also been shown in [14] for ruled manifolds.

To show that the flow exists for all time we first need to show that $\phi_{t}(x)$ does not become zero in finite time for $x \in(0, m)$. Let $\Theta$ be a fixed momentum profile, i.e., a non-negative function on $[0, m]$, strictly positive on the interior, and satisfying the usual boundary conditions. We want to show

Proposition 12. - If $\phi_{t}$ is the solution to the Calabiflow, then $\sup \frac{\Theta(x)}{\phi_{t}(x)}$ does not blow up in finite time.

Proof. - This follows from Lemma 11 and the following lemma.

Lemma 13. - Given a constant $C>0$ there exists a constant $D>0$ such that if for a momentum profile $\psi$ we have

$$
\int_{0}^{m} \frac{\Theta}{\psi} d \tau<C \quad \text { and } \quad\|\psi\|_{C^{1,1 / 2}}<C
$$

then

$$
\sup \Theta / \psi<D
$$


Proof. - Let us derive the estimate near the boundary first. Because of the $C^{1,1 / 2}$ bound on $\psi$, there exists a constant $C_{1}$ such that

$$
\left|\psi^{\prime}(x)-\psi^{\prime}(0)\right|<C_{1} \sqrt{x}
$$

i.e.,

$$
\psi^{\prime}(x)>2-C_{1} \sqrt{x}
$$

This implies that

$$
\psi(x)>x\left(2-\frac{2}{3} C_{1} \sqrt{x}\right),
$$

so that for $x<\left(3 / 2 C_{1}\right)^{2}$ we have $\psi(x)>x$. We can apply the same argument around $x=m$ as well, so we obtain a small constant $\delta$ such that

$$
\text { if } x<\delta \text { or } x>m-\delta \text {, then } \frac{\Theta(x)}{\psi(x)}<D \text {. }
$$

Now we concentrate on the set $(\delta, m-\delta)$. On this set we have a uniform lower bound $\Theta(x)>\epsilon>0$ so we just need a lower bound on $\psi$. There is a constant $C_{2}$ such that $\left|\psi^{\prime}(x)\right|<C_{2}$ for all $x$. Suppose that for some $x \in(\delta, m-\delta)$ we have $\psi(x)<\epsilon / k$ where $k$ is large. Assume for simplicity that $x<m / 2$. Then for $y<m / 2-\delta$ we have

$$
\psi(x+y)<\frac{\epsilon}{k}+C_{2} y \text {. }
$$

Writing $a=m / 2-\delta$, this implies that

$$
C>\int_{0}^{m} \frac{\Theta}{\psi} d \tau>\epsilon \int_{0}^{a} \frac{1}{\frac{\epsilon}{k}+C_{2} y} d y>\frac{\epsilon}{C_{2}}\left[\log C_{2} a-\log \frac{\epsilon}{k}\right] .
$$

Since this tends to infinity as $k \rightarrow \infty$, we get the required lower bound on $\psi(x)$ for $x \in(\delta, m-\delta)$. Combining this with the boundary estimate we obtain the statement of the lemma.

Next we would like to estimate the derivatives of $\phi$ following the calculation in Guan [14]. Let us introduce the functional

$$
L(\phi)=\int_{0}^{m}\left(\phi S(\phi)^{\prime \prime}\right)^{2}(1+\tau) d \tau
$$

We want to show

Lemma 14 (Guan [14]). - For $\phi_{t}$ a solution of the Calabi flow we have that $L\left(\phi_{t}\right) \leqslant C(t)$ for some function $C(t)$ defined for all $t$.

Proof. - All our constants will depend on $t$ but will be finite for all $t$. All the integral norms will be with respect to the measure $d \tau$ and not $(1+\tau) d \tau$ as before.

In the proof we will repeatedly use the Hardy-type inequality

$$
\|f\|_{L^{2}(0, m)} \leqslant C\left\|\phi_{t}^{-k+1}\left(\phi_{t}^{k} f\right)^{\prime}\right\|_{L^{2}(0, m)}
$$

for $k \geqslant 1$ and any $f \in C^{1}[0, m]$ with the constant $C$ depending on $t$. Using Proposition 12, this is easy to derive from the inequality

$$
\int_{-1}^{1} f(x)^{2} d x \leqslant C \int_{-1}^{1}\left[\left(1-x^{2}\right)^{-k+1}\left(\left(1-x^{2}\right)^{k} f(x)\right)^{\prime}\right]^{2} d x .
$$


This in turn follows from the inequality

$$
\int_{0}^{1} f(x)^{2} d x \leqslant C \int_{0}^{1}\left(x^{-k+1}\left(x^{k} f\right)^{\prime}\right)^{2} d x
$$

for $f$ with $f(1)=0$, applied to the intervals $[-1,0]$ and $[0,1]$ separately (see [14]).

Let us compute the derivative of $L\left(\phi_{t}\right)$.

$$
\begin{aligned}
\frac{d}{d t} L\left(\phi_{t}\right) & =2 \int_{0}^{m}\left(\phi_{t} S\left(\phi_{t}\right)^{\prime \prime}\right)^{3}(1+\tau) d \tau-\int_{0}^{m}\left[(1+\tau) \phi_{t}^{2} S\left(\phi_{t}\right)^{\prime \prime}\right]^{\prime \prime 2} \frac{d \tau}{1+\tau} \\
& \leqslant C_{1} \int_{0}^{m}\left(\phi_{t} S\left(\phi_{t}\right)^{\prime \prime}\right)^{3} d \tau-C_{2}\left\|\left(\phi_{t}^{2} S\left(\phi_{t}\right)^{\prime \prime}\right)^{\prime \prime}\right\|_{L^{2}}^{2} .
\end{aligned}
$$

Let us estimate the cubed term. We have

$$
\begin{aligned}
\int_{0}^{m}\left(\phi_{t} S\left(\phi_{t}\right)^{\prime \prime}\right)^{3} d \tau & \leqslant C_{3}\left\|\phi_{t} S\left(\phi_{t}\right)^{\prime \prime}\right\|_{C^{0}} L\left(\phi_{t}\right) \\
& \leqslant C_{4}\left\|\left(\phi_{t} S\left(\phi_{t}\right)^{\prime \prime}\right)^{\prime}\right\|_{L^{2}} L\left(\phi_{t}\right) \\
& \leqslant C(\epsilon) L\left(\phi_{t}\right)^{2}+\epsilon\left\|\left(\phi_{t} S\left(\phi_{t}\right)^{\prime \prime}\right)^{\prime}\right\|_{L^{2}}^{2}
\end{aligned}
$$

for any $\epsilon>0$ using Young's inequality. Using the uniform $H^{2}$-bound on $\phi_{t}$ and the Hardytype inequality twice we obtain

$$
\begin{aligned}
\left\|\left(\phi_{t}^{-1} \cdot \phi_{t}^{2} S\left(\phi_{t}\right)^{\prime \prime}\right)^{\prime}\right\|_{L^{2}} & \leqslant\left\|\phi_{t}^{-1}\left(\phi_{t}^{2} S(\phi)^{\prime \prime}\right)^{\prime}\right\|_{L^{2}}+\left\|\phi_{t}^{\prime} S\left(\phi_{t}\right)^{\prime \prime}\right\|_{L^{2}} \\
& \leqslant C_{5}\left\|\phi_{t}^{-1}\left(\phi_{t}^{2} S\left(\phi_{t}\right)^{\prime \prime}\right)^{\prime}\right\|_{L^{2}} \\
& \leqslant C_{6}\left\|\left(\phi_{t}^{2} S\left(\phi_{t}\right)^{\prime \prime}\right)^{\prime \prime}\right\|_{L^{2}}
\end{aligned}
$$

so if we choose $\epsilon$ small enough (depending on $t$ ), then we obtain the inequality

This implies that

$$
\frac{d}{d t} L\left(\phi_{t}\right) \leqslant C_{1}(t) L\left(\phi_{t}\right)^{2}
$$

i.e., for any $T>0$ we have

$$
\frac{d}{d t} \log L\left(\phi_{t}\right) \leqslant C_{1}(t) L\left(\phi_{t}\right)
$$

$$
\log L\left(\phi_{T}\right) \leqslant \log L\left(\phi_{0}\right)+\sup _{t \in[0, T]} C_{1}(t) \int_{0}^{T} L\left(\phi_{t}\right) d t .
$$

Now Lemma 9 gives a bound on the integral of $L\left(\phi_{t}\right)$ since the Calabi functional is nonnegative, so the proof is complete.

Now we need to use the inequality

$$
\|f\|_{L^{2}}^{2} \leqslant C\left(\left\|\phi f^{\prime}\right\|_{L^{2}}^{2}+f(m / 2)^{2}\right)
$$

for all $f \in C^{1}(0, m)$ which can be proved in the same way as the Hardy-type inequalities we used before. This implies that

$$
\left\|S\left(\phi_{t}\right)\right\|_{C^{0}}^{2} \leqslant C_{1}\left\|S\left(\phi_{t}\right)^{\prime}\right\|_{L^{2}}^{2} \leqslant C_{2}\left[\left\|\phi_{t} S\left(\phi_{t}\right)^{\prime \prime}\right\|_{L^{2}}^{2}+\left(S\left(\phi_{t}\right)^{\prime}(m / 2)\right)^{2}\right] .
$$

The bound on $\left\|\phi_{t} S\left(\phi_{t}\right)^{\prime \prime}\right\|_{L^{2}}$ gives a bound on $\left|S\left(\phi_{t}\right)^{\prime}(x)-S\left(\phi_{t}\right)^{\prime}(m / 2)\right|$ for $x$ inside the interval $\left(\frac{m}{3}, \frac{2 m}{3}\right)$. The bound on $\left\|S\left(\phi_{t}\right)\right\|_{L^{2}}$ (the Calabi functional decreases along the flow) then gives an a priori bound on $S\left(\phi_{t}\right)^{\prime}(m / 2)$. Therefore as long as $L\left(\phi_{t}\right)$ remains bounded, we have a $C^{2}$ bound on $\phi_{t}$ (depending on $t$ ). To obtain estimates for the higher derivatives 
of $\phi_{t}$ we could either continue with similar integral estimates in the manner of [14] or we can note that a $C^{2}$ bound on the momentum profile implies a uniform bound on the Ricci curvature. According to Chen-He [6] the Calabi flow exists for all time as long as the Ricci curvature remains uniformly bounded.

\section{REFERENCES}

[1] V. Apostolov, D. M. J. Calderbank, P. Gauduchon, C. W. TønnesenFriedman, Hamiltonian 2-forms in Kähler geometry. III. Extremal metrics and stability, Invent. Math. 173 (2008), 547-601.

[2] T. Aubin, Équations du type Monge-Ampère sur les variétés kählériennes compactes, Bull. Sci. Math. 102 (1978), 63-95.

[3] E. Calabi, Extremal Kähler metrics, in Seminar on Differential Geometry, Princeton (S. T. Yau, ed.), 1982.

[4] X. Chen, Calabi flow in Riemann surfaces revisited: a new point of view, Int. Math. Res. Not. 2001 (2001), 275-297.

[5] X. Chen, Space of Kähler metrics. III. On the lower bound of the Calabi energy and geodesic distance, Invent. Math. 175 (2009), 453-503.

[6] X. Chen, W. Y. He, On the Calabi flow, Amer. J. Math. 130 (2008), 539-570.

[7] X. Chen, W. Y. He, The Calabi flow on toric Fano surfaces, preprint arXiv:0807.3984.

[8] P. T. Chruściel, Semi-global existence and convergence of solutions of the RobinsonTrautman (2-dimensional Calabi) equation, Comm. Math. Phys. 137 (1991), 289313.

[9] S. K. Donaldson, Remarks on gauge theory, complex geometry and 4-manifold topology, in Fields Medallists' lectures, World Sci. Ser. 20th Century Math. 5, World Sci. Publ., River Edge, NJ, 1997, 384- 403.

[10] S. K. Donaldson, Scalar curvature and stability of toric varieties, J. Differential Geom. 62 (2002), 289-349.

[11] S. K. Donaldson, Conjectures in Kähler geometry, in Strings and geometry, Clay Math. Proc. 3, Amer. Math. Soc., 2004, 71-78.

[12] S. K. Donaldson, Lower bounds on the Calabi functional, J. Differential Geom. 70 (2005), 453-472.

[13] A. FujIKI, Moduli space of polarized algebraic manifolds and Kähler metrics, Sugaku Expositions 5 (1992), 173-191.

[14] D. Guan, Extremal solitons and exponential $C^{\infty}$ convergence of the modified Calabi flow on certain $\mathbb{C P}^{1}$ bundles, Pacific J. Math. 233 (2007), 91-124.

[15] A. D. Hwang, On the Calabi energy of extremal Kähler metrics, Internat. J. Math. 6 (1995), 825-830.

[16] A. D. Hwang, M. A. Singer, A momentum construction for circle-invariant Kähler metrics, Trans. Amer. Math. Soc. 354 (2002), 2285-2325.

[17] T. Mabuchi, Stability of extremal Kähler manifolds, Osaka J. Math. 41 (2004), 563582. 
[18] J. Ross, R. Thomas, An obstruction to the existence of constant scalar curvature Kähler metrics, J. Differential Geom. 72 (2006), 429-466.

[19] N. Sesum, G. Tian, Bounding scalar curvature and diameter along the Kähler Ricci flow (after Perelman), J. Inst. Math. Jussieu 7 (2008), 575-587.

[20] M. Struwe, Curvature flows on surfaces, Ann. Sc. Norm. Super. Pisa Cl. Sci. 1 (2002), 247-274.

[21] G. Székelyhidi, Extremal metrics and $K$-stability, Ph.D. Thesis, Imperial College London, 2006, arXiv:math/0611002.

[22] G. SzÉKelyhidi, Extremal metrics and K-stability, Bull. Lond. Math. Soc. 39 (2007), 76-84.

[23] G. Tian, On Calabi's conjecture for complex surfaces with positive first Chern class, Invent. Math. 101 (1990), 101-172.

[24] G. TIan, Kähler-Einstein metrics with positive scalar curvature, Invent. Math. 130 (1997), 1-37.

[25] C. W. Tønnesen-Friedman, Extremal Kähler metrics on minimal ruled surfaces, $J$. reine angew. Math. 502 (1998), 175-197.

[26] S. T. YAU, On the Ricci curvature of a compact Kähler manifold and the complex Monge-Ampère equation. I, Comm. Pure Appl. Math. 31 (1978), 339-411.

[27] R. YE, The logarithmic Sobolev inequality along the Ricci flow, preprint arXiv:0707.2424, 2007.

[28] Q. S. Zhang, A uniform Sobolev inequality under Ricci flow, Int. Math. Res. Not. IMRN 17 (2007), Art. ID rnm056.

\footnotetext{
Gábor SzÉKELYHIDI

Department of Mathematics

Columbia University 2990 Broadway

New York, NY 10027, U.S.A.

E-mail : gaborsz@gmail.com
} 